Revista Iberoamericana, Vol. LXXIV, Núm. 223, Abril-Junio 2008, 345-359

\title{
NACIÓN Y NARRACIÓN DE LA VIOLENCIA EN COLOMBIA (DE LA HISTORIA A LA SOCIOLOGÍA)
}

POR

\author{
María Helena Rueda \\ Smith College
}

En las últimas décadas, una parte significativa de los textos que se han escrito sobre Colombia, en campos como la literatura, la crónica, la historia y las ciencias sociales, otorgan a la "violencia" un papel central en la configuración de la vida social, política, económica y cultural del país. Las razones de esta recurrencia resultan evidentes. La violencia en Colombia ha tenido efectos catastróficos sobre la configuración social del país y sobre las vidas individuales de la gran mayoría de sus habitantes. ${ }^{1}$ Se trata de una situación de extrema vulnerabilidad, que ha originado una también extrema cantidad de escritura al respecto. Es el resultado de procesos cuyo origen puede remontarse a la época de la Colonia. Sin embargo, la proliferación de estudios y narraciones sobre la violencia es relativamente reciente. En este artículo analizaré de qué manera dicho discurso constituyó una mudanza con respecto a la forma como se refería a los hechos violentos la historiografía heredada del siglo xIx. Dicha mudanza indicaría una “crisis” en las narrativas de nación prevalecientes

\footnotetext{
${ }^{1}$ Cuando se narra el proceso por el cual Colombia ha llegado a tales extremos, se plantean preguntas sobre aquello que es excepcional con respecto a la historia de este país que desde la época de la Independencia ha tenido siempre algún tipo de conflicto armado, de mayor o menor intensidad, en su territorio. Algunos de los estudios más interesantes al respecto coinciden en señalar la forma en que se definieron los vínculos de pertenencia y los antagonismos en el siglo XIX como uno de los factores más determinantes en este sentido. Daniel Pécault (2001) afirma que a partir de mediados del siglo xix las señales de identificación colectiva en Colombia giraban en torno a los dos partidos, liberal y conservador, y no en torno al estado-nación, lo cual habría llevado a una falta de legitimidad en el ejercicio del poder y la violencia por parte del gobierno. Cristina Rojas (2002) plantea que el enfrentamiento entre conservadores y liberales, en los esfuerzos discursivos por definir el camino hacia la "civilización", terminó por construir un sistema simbólico en el cual el exterminio del adversario se entendía como la única opción. Marco Palacios y Frank Safford (2002) señalan que a lo largo de la historia de Colombia los patrones de identificación comunitaria se desarrollaron más con las regiones que con la nación. En general se habla de una comunidad nacional definida en torno a antagonismos irreconciliables, ante los cuales la violencia aparecía como un componente ineludible de la vida social. Esto llevó a un constante estado de guerra que aún se perpetúa. La idea de la "naturalización" de la violencia es también recurrente en las referencias a la situación colombiana. Se utilizan también términos como "normalización” o "banalización” de la violencia, es decir el hecho de que el asesinato y el crimen se han convertido en prácticas cotidianas de la vida social en Colombia. El término "naturalización" es utilizado en la introducción del libro Nuevas visiones sobre la violencia en Colombia (Camacho et al., 1997). Daniel Pécault utiliza la expresión "violencia banalizada”.
} 
durante la modernización, la cual se manifiesta justamente cuando comienza el discurso sobre la "violencia".

El acercamiento a la violencia en la escritura, en cualquier contexto, aparece con frecuencia acompañado de un cierto malestar, tanto por la brutalidad de los eventos observados, como por la incapacidad del texto para remediar aquello que constituye su objeto: hechos irreversibles que siempre preceden y sobrepasan lo que se escribe acerca de ellos. ${ }^{2}$ A esto se suma el que los textos no puedan realmente apartarse de dichos excesos. Si bien la escritura ofrece la posibilidad de un distanciamiento con respecto a la violencia, es difícil pensarla como un acto ajeno a ella. En Colombia, por ejemplo, la tradición de escritura que se inicia en el campo literario con las llamadas "novelas de la violencia”, y en las ciencias sociales con el libro de Orlando Fals Borda, Eduardo Umaña y Germán Guzmán, La violencia en Colombia (1962), el primero de una serie de estudios en la tendencia conocida luego como "violentología”, ${ }^{3}$ es inseparable de los conflictos a los que se refiere, frente a los cuales ha jugado muy diversos papeles. ${ }^{4}$

La escritura sobre hechos violentos se integra con frecuencia en proyectos más amplios de configuración de una comunidad. El discurso historiográfico heredado del siglo xix tendía a pensar la violencia en términos de esfuerzos por consolidar la nación. Hasta bien entrado el siglo xx, dicho discurso se caracteriza por un énfasis en el aspecto heroico de las guerras, sin referencias a la violencia como "problema”. Con la expansión de las ciencias sociales,

\footnotetext{
${ }^{2}$ Frente a esta conciencia, ciertos textos asumen la estrategia de proyectar hacia el futuro la posibilidad de actuar con respecto a los hechos narrados: se ofrecen como un medio para evitar que acciones similares vuelvan a ocurrir, mediante el mecanismo de describir las circunstancias que llevaron a que se presentara la catástrofe, las cuales podrían ser manejadas de otra manera si volvieran a presentarse. ${ }^{3}$ La "violentología" es una denominación que se le dio en Colombia a la práctica de intelectuales de las ciencias sociales que se dedicaban exclusivamente al estudio de la violencia. Tuvo su época de auge con el establecimiento de la Comisión Nacional de Estudios sobre la Violencia, en 1987, y sus trabajos se dieron a conocer por primera vez con la publicación del libro Colombia, violencia y democracia (Arocha et. al., 1987). Gran parte de los esfuerzos de este grupo de investigadores se dirigieron a estudiar el período que va aproximadamente desde 1946 hasta 1966, etapa conocida como La Violencia, en la cual los campos colombianos fueron escenario de numerosas masacres y sucesos de extrema crueldad, por enfrentamientos entre el partido liberal y el conservador. Más adelante en este artículo me referiré a esto con mayor detalle. Para un análisis sobre la evolución intelectual y la significación política y cultural de los "violentólogos", ver el texto de Santiago Villaveces Izquierdo, "The Crossroads of Faith: Heroism and Melancholia in the Colombian 'Violentologists' (1980-2000)” (2006).

${ }^{4} \mathrm{La}$ acción que ejercen los textos sobre la violencia en una sociedad varía según el tipo de escritura. Cierto lenguaje performativo, cuyo más claro exponente es el texto de la Ley, define cuáles actos de violencia son inaceptables en una comunidad, mientras a la vez regula los castigos que se otorgará a quienes incurran en ellos. Se trata de un acto discursivo con claros efectos sobre los cuerpos que conforman una sociedad. Con frecuencia los textos sobre la violencia buscan influir en el campo de la legislación, para tener un efecto de este tipo, y prevenir la violencia de tipo catastrófico. Hay también casos en los que la narración de hechos violentos procura su integración en el terreno de lo social, al sustraerlos cuando menos parcialmente del ámbito de lo inaprensible, y presentarlos como lenguaje. En otras ocasiones, los textos buscan por el contrario reproducir la capacidad de la violencia para quebrar esquemas sociales y producir una conmoción en los mismos. Es el aspecto transgresor de la escritura, que paradójicamente puede constituirse en una alternativa a discursos que naturalizan la violencia.
} 
que en Colombia (como en otros lugares de América Latina) ocurre hacia la década de 1950, se introduce una concepción orgánica de la sociedad y un estudio sistemático de la misma. En ella se trabaja con frecuencia bajo la noción del Estado como instancia encargada de ejercer el monopolio sobre el ejercicio de la violencia, lo cual lleva a pensar la violencia de tipo catastrófico principalmente en términos de fallas en la estructura del Estado. Me ocuparé enseguida de estudiar la evolución de estos discursos en el contexto de los procesos de consolidación nacional en Colombia, y su relación con el papel que en la concepción moderna de la nación juega la representación textual de la violencia.

HISTORIA, NACIÓN Y MUERTE

En su conocido libro sobre el origen y la expansión del nacionalismo, Imagined Communities (1983, 1991), Benedict Anderson otorga un papel central a la cultura de imprenta en el proceso de formación de las naciones modernas. Según el análisis de Anderson, el consenso que lleva a "imaginar" una nación surge en parte de la sensación de simultaneidad que produce el uso de una temporalidad homogénea en periódicos y novelas, lo cual contribuye a difundir en la comunidad nacional la idea de compartir una serie de vivencias, costumbres y tradiciones. En respuesta a la primera edición del libro de Anderson, Homi Bhabha hace en "DissemiNation” (1990) una crítica a la idea de la nación como comunidad nacida de este tipo de consenso, al señalar que la homogeneidad difundida por la cultura de imprenta oculta el aspecto disociador de la misma. Para el caso de América Latina, Ángel Rama había planteado ya en La ciudad letrada (1984) que la cultura letrada contribuyó a la configuración de esquemas sociales excluyentes, por los cuales se garantizaban los beneficios de las élites. Por la escritura se buscó también, sin embargo, la incorporación de grandes contingentes poblacionales (letrados o no) en los diversos proyectos nacionales. El elitismo de la cultura de imprenta iba acompañado por una necesidad de apelar a la colectividad ampliada a la que hacía referencia.

La forma como el discurso de la nación manejaba la aparente oposición entre su afán de incluir una población extensa y su tendencia a crear paradigmas de exclusión está relacionada con el papel que en el proceso de imaginar una nación le cabe a la representación de la violencia. En el primer capítulo de Imagined Communities, Anderson señala que la idea de la nación surge como respuesta de la modernidad al temor que produce la muerte, reemplazando el vacío que en este sentido dejaba la disolución del sentimiento religioso. A través de ella se une secularmente la existencia individual con la de la comunidad nacional, pues la contingencia de la vida humana adquiere sentido al vincularse con el pasado y el futuro de la nación. La pertenencia apela a una sensación de vulnerabilidad frente a la cual la nación se ofrece como entidad protectora. Se verifica así una parte importante del carácter performativo y pedagógico que, de acuerdo con Bhabha, posee el discurso nacional. La nación se ofrece como un amparo contra la violencia, y a la vez legitima la violencia ejercida en nombre de ella. Al estar basada en el relato de una tradición compartida, además, la idea de la nación se concentra en una violencia situada siempre en el pasado, olvidada en el proceso mismo de evocarla.

En L'écriture de l'histoire (1975), Michel de Certeau señala que la historiografía moderna comienza con el establecimiento de una disociación entre las nociones de pasado y 
presente, por la cual el primer elemento de dicha oposición es definido como un vacío sobre el cual el historiador escribe su texto, asumiendo la tarea de dar voz a los muertos de una comunidad. El discurso de la modernidad se separa así de la tradición religiosa, manteniendo con ella una relación de deuda y rechazo a la vez. El trabajo de la historia consiste en "hacer hablar" al cuerpo social, con respecto al cual establece sin embargo una distancia, pues lo asume como "cuerpo muerto", inhabilitado para hablar. Los habitantes de la comunidad son "hablados" por un discurso histórico que los interpela desde sus muertos y desde la vulnerabilidad que éstos evocan. La violencia del cuerpo hace así posible la escritura de la historia, pero sólo como ausencia. El discurso occidental mantiene con respecto a ella una actitud dual de fascinación y distanciamiento, pues la requiere para poder hablar pero al hablar pretende superarla. Esto se relaciona también con la idea de la tradición nacional como olvido colectivo de la violencia, que aparece en el libro de Benedict Anderson.

En Imagined Communities, Anderson analiza la necesidad del olvido en las historias nacionales a partir de la lectura de dos frases tomadas del texto Qu'est-ce qu'une nation?, escrito por Ernest Renan en 1882. Dicho olvido se refiere específicamente a los hechos de violencia que se consideran fundadores de la comunidad nacional. La primera frase se refiere al olvido colectivo como elemento inherente a la idea de la nación: “Or, l'essence d'une nation est que tous les individus aient beaucoup de choses en commun et aussi que tous aient oublié bien des choses” (199) (“Así, la esencia de una nación es que todos los individuos tengan muchas cosas en común y también que todos hayan olvidado muchas cosas”). La segunda problematiza esta referencia al señalar que ese olvido se dirige precisamente a hechos de violencia ocurridos en el pasado: "Tout citoyen français doit avoir oublié la Saint-Barthélemy, les massacres du Midi au XIIIe siècle” (199) ("Todo ciudadano francés debe haber olvidado la Saint-Barthélemy, las masacres de Midi en el siglo XIII”). Según Anderson, los eventos y personajes relacionados con esos episodios de gran violencia ubicados en un pasado remoto serían evocados en la historia de las naciones como algo que toda la nación debe recordar, sólo para compartir el hecho de haberlo olvidado colectivamente. La no resolución de la dualidad olvido/recuerdo sustenta el discurso que define la comunidad nacional.

Qu'est-ce qu'une nation? ha sido uno de los textos más utilizados por la teoría occidental para reflexionar sobre la nación y el nacionalismo en cuanto nociones modernas. ${ }^{5}$ Renan se refiere allí específicamente al origen violento de la nación y al hecho de que el relato histórico cumple la función de representar esa violencia: "L'investigation historique, en effet, remet en lumière les faits de violence qui se sont passés à l’origine de toutes les formations politiques" ("La investigación histórica, en efecto, saca a la luz aquellos hechos de violencia que han ocurrido en el origen de todas las formaciones políticas”). Según la definición de Renan, la nación, para ser pensada como tal, requiere no sólo que hayan existido esas muertes violentas sino sobre todo que éstas sean relatadas desde el olvido, olvidadas en el momento mismo en que son evocadas en la historia nacional. Esto porque en el gran relato de la nación conviene que ésta sea presentada como resultado de un duelo colectivo: "En

\footnotetext{
${ }^{5}$ Entre los autores que han utilizado el texto de Renan como base para sus reflexiones sobre la nación y el nacionalismo Homi Bhabha señala a Eugene Kamenka, Ernest Gellner, Tzvetan Todorov y el propio Benedict Anderson.
} 
fait de souvenirs nationaux, les deuils valent mieux que les triomphes, car ils imposent des devoirs, ils commandent l'effort en común” (“En lo referente a recuerdos nacionales, los duelos son más útiles que los triunfos, porque imponen deberes, impulsan el esfuerzo común”). Dicho duelo colectivo es el resultado de una representación histórica que permite a la nación funcionar como entidad política y económica en la modernidad.

HACIA LA NACIÓN ESCINDIDA:

EL RELATO DE LA HISTORIA EN COLOMBIA

Las características señaladas por el texto de Renan para el discurso histórico nacional son visibles en textos de historia hispanoamericanos de finales del siglo xIX y principios del Xx, una época de enfrentamientos políticos y militares en prácticamente todas las repúblicas del continente, durante la cual los historiadores buscaban contribuir a la configuración de una idea de nación que facilitara los procesos de modernización. Germán Colmenares (1987) señala que por esta razón la historiografía de las repúblicas hispanoamericanas durante el siglo XIX estuvo dedicada en su mayor parte a relatar los hechos ocurridos durante la Independencia. Los historiadores se sentían herederos de las guerras revolucionarias y asumían su labor como un proceso con el cual ayudaban a construir la nación. Esto contribuía también a ofrecer una cierta racionalidad y sentido a la violencia que seguía siendo parte de la vida cotidiana de las repúblicas en todo el siglo xix. Se la entendía justificada por el mismo fin trascendente que había impulsado la causa independentista: es decir la defensa de la causa nacional.

Colombia no es una excepción en este sentido. Como en otros países latinoamericanos, la historiografía decimonónica se concentró allí principalmente en investigar los sucesos de la Independencia. La obra más influyente en ese sentido fue la Historia de la Revolución, en la República de Colombia (1858), de José Manuel Restrepo, publicada primero en Paris en 1827 y luego en Besanzón en 1854, en una edición muy aumentada que se considera definitiva. El autor había participado en la campaña independentista, conocía personalmente a algunas de sus figuras más destacadas y formaba parte del gobierno republicano. Se mostraba entusiasta con la causa de la revolución y definía la ruptura con España como un hecho “justo y necesario" para la patria. Es una obra extensa y minuciosa en su recuento de los combates y los hechos políticos que los acompañaron, de manera que aún hoy constituye una de las fuentes de investigación histórica más importantes sobre el período de la Independencia. Constituyó la base principal de los manuales de historia para la enseñanza en las escuelas, que se comenzarían a escribir durante la Regeneración, una etapa iniciada cuando subió al poder Rafael Núñez, quien fue presidente cuatro veces entre 1880 y 1892, gobernando con las banderas del orden, la unidad nacional y la pacificación. ${ }^{6}$

\footnotetext{
${ }^{6}$ El primer manual escrito específicamente con este propósito durante la Regeneración fue el de Constancio Franco, llamado Compendio de la historia de la revolución de Colombia para el uso de las escuelas (1881), el cual fue publicado con una carta del presidente Núñez que avalaba la edición y con un prólogo de él mismo en el cual elogiaba los aspectos moralizantes de la obra. Se dedicaba exclusivamente al estudio de la etapa de la Independencia, definiéndola como una revolución justa y buena. Hacía especial énfasis en la descripción de las virtudes morales de los próceres, definiéndoles como héroes que servían de ejemplo a la población de la república.
} 
La prioridad de Núñez consistió en fortalecer el Estado y establecer un régimen proteccionista, durante una etapa marcada por la quiebra de las exportaciones colombianas, principalmente el café, en los mercados internacionales. ${ }^{7}$ Para ello fomentó una moralización de las ideas y las costumbres, la cual fue emprendida por intermedio de una alianza con los conservadores y con la adopción de la religión católica como fuerza unificadora de la nación. Cuando llegó al poder, el país se regía por una constitución proclamada por los liberales en 1863, la cual era promotora del libre cambio, federalista y anticlerical. Su extremo afán reformista y la violenta oposición al mismo que ejercieron los sectores conservadores, junto a factores económicos relacionados con la caída de las exportaciones, habían dejado una secuela de inestabilidad y guerra en todo el territorio nacional colombiano. Núñez se designó como el encargado de "reordenar" el país, denominando su régimen con el término "regeneración”, asociado a la moralización de los colombianos. Dicho proceso implicó una redefinición de paradigmas nacionales, que se llevó a cabo en gran parte por la reescritura de textos como la constitución y los libros de historia. ${ }^{8}$ Esto vino acompañado de un proceso de difusión del patriotismo a través del sistema educativo nacional, que se consolidó también en esa época, gracias a una alianza con la Iglesia católica.

En 1886 Núñez proclamó una nueva constitución, redactada por el conservador Miguel Antonio Caro, en la cual la república quedaba consagrada al Sagrado Corazón y se subordinaban sus mandatos a los de la Iglesia católica, mediante la inclusión de un pacto con la Santa Sede. El centralismo se convirtió en el nuevo sistema de gobierno y la instrucción pública fue entregada a la Iglesia. La labor historiográfica comenzó a centrarse en la escritura de una historia nacional que cumpliera una función ejemplarizante, de apego a la causa de la nación y fomento de virtudes morales como la abnegación y la generosidad. Este empeño conduciría a la conformación de la Academia Colombiana de Historia en 1902, una de cuyas labores fue la difusión de la historia nacional en las escuelas, como medio para lograr la “moralización” del país. Para el centenario de la Declaración de Independencia, en 1910, se realizó un concurso con el objeto de elaborar un manual de historia patria destinada a la instrucción pública, en el cual resultaría elegido el libro de Jesús María Henao y Gerardo Arrubla, Historia de Colombia para la enseñanza secundaria (1911), usado extensamente como texto pedagógico hasta finales de la década de 1970.

El énfasis moralizador de esta obra queda claro desde el prólogo, donde los autores declaran que la enseñanza de la historia "contribuye a la formación del carácter, moraliza, aviva el patriotismo y prepara con el conocimiento de lo que fue a la activa participación

\footnotetext{
${ }^{7}$ En 1875 el precio de la libra de café en Nueva York cayó de 20.5 a 10.5 centavos la libra. Entre 1879 y 1881 el precio de la quina bajó en un $80 \%$. Las exportaciones en general en Colombia bajaron de 29 millones de dólares en 1875 a 7.3 millones en 1885. Las quiebras que esto trajo consigo vinieron acompañadas de varias guerras civiles que dejaron al país en un estado de crisis que condujo al surgimiento de un régimen como el de la Regeneración. Estas cifras están tomadas del estudio de Jorge Orlando Melo, “La evolución económica de Colombia, 1830-1900” (1979).

${ }^{8}$ También durante aquellos años, en 1887, se adoptó el himno nacional de Colombia, un largo poema redactado por el propio Rafael Núñez, en el que predominan las referencias a la imaginería católica y la alabanza de comportamientos heroicos, en especial los de personajes históricos, como Antonio Ricaurte, descritos con un carácter de mártires.
} 
en el presente” (1: 3-4). ${ }^{9}$ En dicho proyecto, toda la narración de hechos de violencia está marcada por un afán ejemplarizante, de manera que el texto se concentra principalmente en el relato de hazañas heroicas, y en la descripción de quienes las realizaron. En este sentido no difiere en gran medida del discurso historiográfico que prevaleció en toda América Latina durante el siglo XIx, con su énfasis en la exaltación de los "grandes hombres" de la vida nacional. Germán Colmenares (1984) señala que con esto se promovía la enseñanza de la historia, mediante la creación de figuras mitificadas que facilitarían la identificación de la población con los paradigmas de la nación. Según Colmenares, este modelo de escritura histórica responde en parte a la lectura de teorías historiográficas europeas de la época y en especial a las ideas de Carlyle en On Heroes, Hero-Worship and the Heroic in History (1841) con respecto a los "grandes hombres" como conductores de la historia.

En dicha visión heroica los hechos violentos no son percibidos como un problema social en sí mismos. Se habla de guerras, matanzas y revoluciones, pero no de "violencia”, como tal. La idea de la nación recoge y contiene los actos de agresión, sin que aparezca una noción de víctimas o victimarios. Los hechos de violencia, por el contrario, proveen el impulso que alienta la construcción de la nación. Al concluir la obra, los autores declaran: “Tenemos fe en que la República se encamina resueltamente a un porvenir de ventura [...] y en que sus hijos, en especial los jóvenes, cultivando el amor del suelo sagrado, aprenderán a apreciar cuánto ha costado y cómo debemos conservarlo y defenderlo” (2: 632). En esta construcción discursiva, la agresión y el sufrimiento de la violencia aparecen como el “costo” que se debió pagar para defender la nación en cuanto entidad "sagrada”, con la cual existen vínculos de parentesco y de "amor”. Es un discurso, sin embargo, que sólo funciona en su referencia a las violencias de un pasado situado en la distancia. La Guerra de los Mil Días, por ejemplo, que tuvo lugar pocos años antes de la época en que se escribió este libro, queda fuera de él. ${ }^{10}$

NARRAR LA VIOLENCIA DEL PRESENTE

Concentrada en narrar las guerras del pasado, la tradición historiográfica heredada del siglo xIX se convertiría entonces en un espacio de salvaguardia para una historia nacional ritualizada, en la cual la época de la Independencia era representada como una etapa de violencia fundacional y las guerras del siglo xıx como conflictos resueltos durante la época de la Regeneración. Más o menos hasta la década de 1950 las agresiones y heridas de las guerras que tuvieron lugar en Colombia a partir de los primeros años del siglo xx no entraban en el discurso historiográfico, aunque sí en crónicas y en relatos novelados. Las obras de tipo novelesco se multiplicaron tras la etapa conocida como La Violencia, una lucha entre diversos grupos armados -algunos afiliados al estado y otros enfrentados a éste-

\footnotetext{
${ }^{9}$ Mis citas del libro se refieren a la siguiente edición: Jesús María Henao y Gerardo Arrubla, Historia de Colombia para la enseñanza secundaria, 2 tomos. Bogotá: Escuela Tipográfica Salesiana, 1912. El libro se siguió editando regularmente, con cambios y ampliaciones, hasta la década de 1970.

${ }^{10}$ La Guerra de los Mil Días se inició en 1899 con una rebelión por parte de los liberales contra el régimen conservador que estaba en el poder. Involucró guerrillas y un recién organizado ejército nacional, dejando un saldo de unos 100.000 muertos hasta su conclusión en 1903. En la primera edición del libro de Henao y Arrubla no se incluye ninguna referencia a ella.
} 
que entre finales de la década de 1940 y principios de la de 1960 dejó aproximadamente 200.000 muertos. $^{11}$

Los enfrentamientos más intensos de La Violencia comenzaron cuando el candidato del partido conservador, Mariano Ospina Pérez, fue elegido presidente en 1946, por una división interna del Partido Liberal que había estado en el poder desde 1930 y contaba con el apoyo de la mayor parte de la población del país. Los liberales perdieron frente al partido minoritario, por haberse presentado a la contienda con dos candidatos, Gabriel Turbay, representante de los liberales moderados que promovían la industria y el comercio, y Jorge Eliécer Gaitán, partidario de la reforma agraria y defensor de las causas de la clase obrera. Muchos cambios legislativos que se habían realizado durante los años en que estuvieron en el poder los liberales fueron desmontados por el gobierno conservador, lo cual contribuyó a incrementar entre los liberales un descontento que se desataría cuando en 1948 Jorge Eliécer Gaitán fue asesinado en Bogotá.

Pese a haber comenzado como un enfrentamiento partidista, la guerra terminó siendo una lucha generalizada en la cual cualquier habitante de las regiones afectadas podía ser víctima. Tanto conservadores como liberales llevaron a cabo campañas de terror para promover la anexión de grandes territorios, mediante métodos de exterminio que buscaban la expulsión de los miembros del partido contrario, quienes huían de sus tierras acosados por el miedo. En los asaltos que se llevaban a cabo se realizaron prácticas exhibicionistas y elaboradas de la agresión, que incluyeron el uso de diversos tipos de “cortes” y la exposición de los cuerpos violentados como trofeos de guerra. ${ }^{12}$ Algunas de estas formas de violencia habían comenzado a ser utilizadas a principios de siglo, durante la Guerra de los Mil Días, y en estos años fueron llevadas al extremo.

Se trata de un momento en la historia de Colombia sobre el cual aún no existe una versión definitiva. Una de las razones es que algunos grupos guerrilleros surgidos durante esa época continuaron activos y constituyeron la base de la cual surgieron las grandes guerrillas que a finales de los años noventa se fortalecieron y revivieron la guerra generalizada en el país, en un enfrentamiento con el ejército y los grupos paramilitares ahora intensificado por la presencia del dinero del narcotráfico. Esto genera la noción de que el conflicto iniciado en ese entonces aún no ha concluido y por ello no puede ser narrado como "historia", es decir como algo que pertenece al pasado. Una percepción común al respecto hoy es que, aunque el acuerdo del Frente Nacional constituyó la conclusión “oficial” de La Violencia,

\footnotetext{
${ }^{11}$ Tanto las fechas como el número de muertos ocurridos durante La Violencia son aún motivo de controversia. Como fechas claves para el comienzo y final del conflicto se mencionan normalmente el asesinato del líder liberal Jorge Eliécer Gaitán en 1948 y la firma del acuerdo entre liberales y conservadores que daría origen al Frente Nacional, en 1957. Sin embargo, prácticamente todos los historiadores coinciden en afirmar que los enfrentamientos habían comenzado antes de la muerte de Gaitán y continuaron varios años después de que se hubiera firmado el mencionado acuerdo. El número de muertes ha sido inflado por unos y disminuido por otros: la cifra de 200.000 corresponde a un término medio entre ambas tendencias y es hoy aceptada como indicativa por muchos expertos.

${ }^{12}$ Por "corte" se entendía el tipo de herida que se propinaba a la víctima para descuartizarlo después de morir. El catálogo de los cortes que fueron utilizados durante La Violencia es macabro y ha sido analizado por varios autores, entre ellos Gonzalo Sánchez y María Victoria Uribe.
} 
los conflictos que le dieron origen no terminaron con ese pacto, el cual fue una mera alianza entre los dirigentes de ambos partidos.

Los campesinos conservadores y liberales que habían asumido la lucha entre los partidos como una causa propia, quedaron en la mayor parte de los casos en una situación precaria. Despojados de sus tierras y cargando el dolor de la guerra, pasaron a dilatar los cordones de miseria en las ciudades o a adentrarse en las zonas de colonización donde más tarde se extenderían los cultivos ilícitos. Se dificultaron además los caminos de participación política, puesto que el acuerdo entre los dos partidos determinaba que se alternarían el poder cada cuatro años, mediante la postulación de un candidato único a la presidencia. Los ciudadanos no podían sentirse representados por unos partidos que ya no se ofrecían como una opción política, sino como la supuesta solución a la violencia que ellos mismos habían promovido.

Aunque después de un período en el que asumieron el poder los militares (195458) se comenzaron a realizar elecciones y las fuerzas militares del Estado dejaron de utilizar abiertamente la agresión contra la población civil, la violencia y el asesinato indiscriminado continuaron. ${ }^{13}$ En esas circunstancias el discurso histórico sobre la realidad nacional difícilmente podía referirse a lo que ocurría en el territorio colombiano. Incluso la palabra "guerra" parecía inapropiada para hablar de un conflicto en el que era evidente que no se estaba combatiendo por una causa "nacional”, y por eso esta etapa terminó por denominarse simplemente La Violencia, un apelativo que se extendería para describir numerosos enfrentamientos cuyo origen y derivaciones se situaban en terrenos sombríos, o ensombrecidos por intereses camuflados. Comenzaron a surgir nuevos discursos textuales sobre lo ocurrido, despegados de la proyección nacional, sublimada característica de la historiografía decimonónica.

Existe una amplia producción discursiva sobre esta etapa de la historia de Colombia, como señalé más arriba. Los primeros textos al respecto tomaron la forma de crónicas o novelas, y más adelante se ocuparon de ello intelectuales formados en campos de las ciencias sociales. A partir de los años cincuenta se produjeron tantas novelas con esta temática que la crítica literaria habla de un ciclo de "Novelas de La Violencia”, al cual se vinculan algunos de los autores más canonizados de la literatura colombiana. Buena parte de estos relatos interpelan al lector mediante la cruda descripción de los actos sangrientos que eran comunes durante aquellos años en Colombia. En algunas se habla sobre los fines personales que perseguían quienes cometían estos crímenes, pero en general aparecen más como el producto de una especie de barbarie colectiva que se habría apoderado de la nación, y principalmente de los miembros del partido opuesto a aquel al que pertenecía el autor. Prácticamente todas las novelas de este ciclo son brutales en su descripción de los horrores, se declaran basadas en hechos reales, y presentan a los autores de los crímenes como bárbaros y a sus opositores como víctimas inocentes, que si acaso incurren en la violencia es sólo por un afán justificado de venganza.

\footnotetext{
${ }^{13}$ Muchos guerrilleros no se acogieron a las amnistías y pactos realizados tras la firma del Frente Nacional. Algunos de ellos se convirtieron en bandoleros, otros formaron nuevas guerrillas, como las FARC, que actualmente incluyen unos 60.000 combatientes enfrentados al ejército nacional y a fuerzas paramilitares.
} 
El primer recuento literario exhaustivo al respecto lo realizó Gerardo Suárez Rondón, quien en 1966 publicó el libro La novela sobre la violencia en Colombia. Dicho estudio se ocupa de cuarenta novelas pertenecientes a este ciclo, un número que no incluye las numerosas obras que se publicaron más tarde sobre esta misma temática. La mayor parte de estas novelas han sido olvidadas, pero algunos autores inicialmente clasificados en este ciclo son bien conocidos, entre ellos Gabriel García Márquez. Otros son menos famosos fuera de Colombia, pero allí son autores canonizados cuyas obras se siguen editando, como Eduardo Caballero Calderón, Hernando Téllez, Manuel Mejía Vallejo y Manuel Zapata Olivella. Algunos han dejado ya de ser editados, pero en su momento gozaron de mucha popularidad y se publicaron varias ediciones de sus libros, entre ellos Daniel Caicedo, Fernando Ponce de León, Eduardo Santa y Jorge Zalamea.

La proliferación de estudios al respecto desde las ciencias sociales comienza a mediados de la década de 1960. Esto es en parte el resultado de la aparición en la escena intelectual de profesionales que se reunieron en torno a la Facultad de Sociología de la Universidad Nacional de Bogotá, creada en 1959, pero responde también a un deseo generalizado entre la población de saber más sobre los sucesos de aquella época. Mientras que el dolor y los resentimientos heredados de La Violencia marcaban la vida de prácticamente todos los colombianos, el discurso oficial declaraba la desaparición de los conflictos y la convivencia pacífica entre los sectores antes enfrentados. El acuerdo entre dirigentes conservadores y liberales fue acompañado de un pacto de silencio sobre lo ocurrido, el cual era contradicho por las escenas de horror que narraban las novelas y las historias que circulaban oralmente sobre los actos violentos realizados.

\section{LA PERSPECTIVA DE LAS CIENCIAS SOCIALES}

El primer estudio sociológico extenso que se publicó en Colombia sobre aquella etapa, $L a$ violencia en Colombia, de Germán Guzmán Campos, Orlando Fals Borda y Eduardo Umaña Luna, se basó no sólo en documentos oficiales sino también en testimonios recogidos por sus autores, en textos utilitarios como cartas y panfletos, y en las novelas que se ocupaban del tema. La tendencia a trabajar desde la recolección de material auténtico se explica por la expansión en el uso de los métodos de las ciencias sociales, con su énfasis en el trabajo de campo y la compilación de datos en el terreno. Acudieron a las novelas porque la mayoría de ellas declaraban estar basadas en hechos reales. El resultado fue un texto en el que la tendencia a la sistematización que caracteriza a las ciencias sociales es matizada por largas citas donde se incluyen fragmentos de las novelas, los cuales usan extensamente varios recursos propios de la literatura, como la metáfora y la narración en primera persona.

En el prólogo a este libro, que inició una determinada forma de pensar la realidad en el país, Orlando Fals Borda revela una actitud similar a la de los historiadores del XIX, en lo que respecta a la importancia de narrar el pasado violento como medio para consolidar la comunidad y proyectarla hacia el futuro, con una serie de responsabilidades y deberes compartidos. La gran diferencia aquí consiste en que el tema de la violencia se convierte en el punto central de discusión. Este hecho impone a la reflexión un tono catastrófico y urgente, en el cual los hechos sangrientos no son ya percibidos como algo que forma parte 
de los esfuerzos de consolidación nacional, sino como un problema, algo que simplemente no debería haber ocurrido. En los primeros párrafos de la obra leemos:

Para la sociedad colombiana, el problema de la “violencia” es un hecho protuberante. Muchos lo consideran como el más grave peligro que haya corrido la nacionalidad. Es algo que no puede ignorarse, porque irrumpió con machetes y genocidios, bajo la égida de guerrilleros con sonoros sobrenombres, en la historia que aprenderán nuestros hijos; porque su huella será indeleble en la memoria de los sobrevivientes y sus efectos tangibles en la estructuración, conducta e imagen del pueblo de Colombia. (1: 12)

En estas frases se revela un cambio radical con respecto a la actitud de la historiografía heredada de la tradición decimonónica. Si en la clásica concepción de Renan los hechos de violencia deben ser recordados desde el olvido para que la nacionalidad pueda proyectarse hacia el futuro, en este caso no se trata ya de evocarlos para olvidarlos sino de enfrentarlos como algo vivo e indeleble. Aunque se habla de esos hechos en pasado, se los está actualizando y considerando como parte del presente, como algo que tiene "efectos tangibles en la estructuración, conducta e imagen del pueblo". La elaboración de un conocimiento factual y activo sobre la violencia se constituye en uno de los elementos por los cuales se define la comunidad.

El hecho de que el estudio que condujo a la realización de este libro haya sido financiado por la Presidencia de la República parece paradójico, puesto que el estudio señalaba claramente la responsabilidad que tuvieron en las matanzas los partidos políticos que se encontraban en el poder, pero en realidad no hace más que corroborar las dificultades que trajo consigo el establecimiento del Frente Nacional, en cuanto a la legitimación del gobierno frente a los ciudadanos. La financiación gubernamental de proyectos investigativos cuyo objeto es sacar a la luz abusos cometidos por gobernantes anteriores ha sido común en muchos países. Por lo general el objeto de estos esfuerzos es revelar "la verdad" sobre dichos hechos, de tal manera que quienes se encuentran en el poder establezcan una distancia frente al régimen anterior, precisamente a través de esas investigaciones. En el caso colombiano, el gobierno que impulsa la investigación sobre lo ocurrido durante La Violencia, heredero del Frente Nacional y de los conflictos partidistas que originaron las masacres, no puede presentarse como desvinculado de ellos. Por eso el ciclo de violencia analizado en este libro no aparece como un proceso de lucha destinado a tener un fin tras el cual la nación colombiana alcanzará la paz, sino como un peligro que está siempre al acecho.

IMAGINACIÓN NACIONAL DE LA VIOLENCIA

La selección de fuentes por parte de los autores de La violencia en Colombia responde en general a un esfuerzo por conocer y relatar lo ocurrido durante los años de La Violencia desde el saber que poseen al respecto aquellas personas y comunidades que vivieron sus efectos en forma más directa, como víctimas o como agresores. En este proceso se lleva a cabo un cambio en el lugar donde los escritores ubican el origen de la "verdad" acerca de lo ocurrido, declarándose en la posición "neutral” de compiladores y presentadores de información. Si anteriormente la credibilidad acerca de lo narrado estaba garantizada por la 
erudición de los autores, ahora este papel es trasladado al campo de la experiencia "real”, de tal manera que quienes han vivido los hechos se convierten en los nuevos poseedores de la "verdad" sobre ellos. Así, los autores por un lado otorgan un valor de autoridad a la experiencia, lo cual permite que se escuchen en el texto voces que antes no tenían acceso a éste, pero por otro integran dichas voces al análisis sociológico, subordinándolas al sistema que construyen a partir de él.

Muchos ejemplos ilustrativos del análisis aparecen en forma de citas tomadas de las múltiples novelas y crónicas de La Violencia que se publicaron durante los años cincuenta y comienzos de los sesenta. Aquellos libros se caracterizaban por su uso de la narración con propósitos ideológicos, de tal manera que si el autor defendía la causa liberal todos los asesinos eran conservadores y viceversa. Uno de estos libros, citado con frecuencia en la obra de Guzmán, Fals y Umaña, es El basilisco en acción (1953), escrito por un autor conservador que utiliza el seudónimo Testis Fidelis. El propósito declarado de dicho libro es defender la imposición de la pena de muerte en Colombia, para lo cual el autor hace una larga enumeración de todos los crímenes que requieren "reparación moral” por medio de este mecanismo. Cita únicamente crímenes cometidos por los guerrilleros liberales, a quienes llama "bandoleros”, como era común entre los conservadores de esa época.

Para dar una idea del tono utilizado en estas obras y de la forma como las historias narradas en ellas son incorporadas por los autores de La violencia en Colombia, voy a transcribir un fragmento de este último libro, en el que se cita El basilisco en acción. Aparece en la primera parte del libro, "Historia y geografía de la violencia”, donde los autores hacen un recuento sobre los conflictos en las diferentes regiones de Colombia:

\footnotetext{
De una carta pastoral de Monseñor Miguel Ángel Builes, obispo de Santa Rosa de Osos, transcribimos algunos apartes que indican a las claras el extremo que se alcanzó en Antioquia:

"Una madre joven de nuestra diócesis, medio enloquecida después de ver partir en trozos a su esposo y a sus tres hijos mayorcitos, cuando acometieron contra el más pequeño de ellos, por su amor materno reaccionó y se abalanzó contra el verdugo, a quien hirió en un brazo. Entonces los once bandoleros restantes cayeron sobre la valiente mujer y la desollaron viva desde la cabeza hasta los pies, y ya de día la arrojaron viva y sanguinolenta a la huerta de la casa, a la acción del sol, de las moscas y animales carnívoros, hasta morir”. (1: 93)
}

Con su énfasis en la descripción de agresiones físicas extremas, la deshumanización de los victimarios y el intenso sufrimiento de la víctima, esta cita constituye un buen ejemplo de cómo circulaban las historias sobre hechos de La Violencia en las múltiples novelas y crónicas que se escribieron al respecto durante los años cincuenta y principios de los sesenta. Su uso como muestra ilustrativa no causa extrañeza en ese sentido. Sorprende, sin embargo, la forma como los autores de La violencia en Colombia integran la anécdota en su análisis, sin cuestionar su veracidad o señalar los filtros a través de los cuales llega hasta ellos: Monseñor Builes y ese autor que se presenta como Testis Fidelis. No incluyen datos sobre cómo conoció la historia el autor de la carta ni pruebas de que esa persona se refiere a hechos reales. No ofrecen información sobre el libro del cual está tomada la cita y sus condicionantes. El hecho violento en sí y el sufrimiento que causó se convierte en un dato factual que se integra en el sistema sociológico. 
Las corrientes dominantes de las ciencias sociales promueven la afirmación del “dato duro” sobre los elementos inconsistentes de la información recogida. Esto en gran parte porque las ciencias sociales, derivadas de la sociología definida por Auguste Comte, están marcadas por sus vínculos con la noción moderna de “progreso”. De ahí su tendencia a privilegiar la elaboración de conocimientos que puedan ser utilizados para determinar “mejoras” en la organización social. La producción de este saber “utilizable” sólo es posible mediante la construcción de un sistema con patrones que puedan ser manipulados. Para poder llevar a cabo esta sistematización, se debe primero definir un campo que es preciso sistematizar y organizar, es decir, una exterioridad que en el proceso de sistematización será declarada como carente de orden en sí misma. Las fuentes primarias utilizadas por las ciencias sociales se sitúan en el terreno asignado a esa exterioridad. De esta manera son incorporadas las "voces” ligadas al campo de la experiencia en el libro de Guzmán, Fals Borda y Umaña Luna, y la "violencia” aparece en general como parte del “desorden” que las caracteriza.

El objetivo último de los autores es señalar un remedio para el problema de la violencia, definido como el principal "mal” que padece la sociedad en conjunto. La sociedad es pensada como un "organismo" y las metáforas médicas son utilizadas con frecuencia en este libro. La introducción al segundo tomo de la obra habla de la violencia como un “cáncer” (2: 10) que padece la sociedad y que es necesario curar. Los autores asumen el papel de científicos que estudian la sociedad como un cuerpo, descubren su enfermedad e indican un posible remedio para la misma. Al final del libro incluyen una extensa sección titulada "En busca de una terapéutica”, la cual emite una serie de "medidas” que según los autores deben ser tomadas para que Colombia se cure de esa "enfermedad”. Esta idea de la "curación” implica una invocación del Estado, como entidad que tiene la capacidad para manipular mecanismos de configuración social.

Parte de las dificultades para pensar esta presencia de la violencia en lo social se relaciona también con la forma conflictiva que asume el concepto de "nación”. Hay en el texto una contradicción no resuelta en la idea de la nación como "solución” a la violencia, y como una entidad en cuyo nombre se justifica el ejercicio de la violencia. Entre los puntos que se señalan en el capítulo de La violencia en Colombia dedicado a describir una "terapia” para el problema estudiado se encuentran los siguientes:

A) Es necesario crear de nuevo en los colombianos "un pensamiento, un interés y una voluntad de nación”. Mientras tal cosa no se realice como logro colectivo, es previsible que perduren factores desintegrantes con sorpresivos afloramientos de violencia.

B) Esto mismo nos lleva a la urgencia de comunicar al pueblo una dinámica conciencia histórica, porque mientras de ella carezca será horda, con todas las regresiones de la horda. (2: 442-443)

El concepto denación aparece en esta afirmación con varias connotaciones problemáticas. En primer lugar se presenta como una noción que existía antes de que comenzaran las matanzas y se perdió a causa de ellas. En segundo lugar es aludida como un elemento opuesto a la violencia, la cual se relaciona con "factores desintegrantes”. En tercer lugar, la nación es definida como opuesta al concepto de "horda", denominación relacionada con fuerzas regresivas, incompatibles con el "progreso”. 
Sabemos que la época de La Violencia no constituyó una ruptura con respecto a una etapa anterior en la cual la nación estaba ya consolidada, por ello la idea de "crear de nuevo" una idea de nación resulta paradójica. Lo ocurrido durante aquella época se relaciona con conflictos acumulados durante años, los cuales tienen que ver precisamente con el hecho de que los habitantes de Colombia podrían no haber desarrollado nunca un compromiso real con la idea de pertenecer a una misma "nación". El proceso de consolidación nacional en Colombia, como otros en América Latina, se realizó a través de una dinámica que desintegraba mientras buscaba la integración, una dinámica relacionada con la idea decimonónica de combatir el barbarismo con la civilización, en la cual los esfuerzos civilizadores conllevaron frecuentemente más "violencia” que la barbarie que buscaban controlar. Lo que este libro invoca no es una voluntad perdida de "nación”, sino tal vez una idea de nación que sólo existió como construcción discursiva. Así, al hablar de violencia se refiere a la crisis de un concepto incapaz de contener y justificar lo ocurrido.

Los ecos del discurso civilizador se escuchan aún en la afirmación de que la "horda" puede ser convertida en nación mediante la creación de una "conciencia histórica”. Esto implica que los despojos y agresiones que sufren quienes entran en la clasificación de "horda" quedarían normalizados en la idea de la nación. La propuesta terapéutica de este libro no señala la violencia implícita en este proyecto y sin embargo la evoca incansablemente, en su minucioso recuento de episodios trágicos, en su constante señalamiento de responsables e incluso en las macabras fotografías que acompañan el texto: un desfile de cuerpos muertos y mutilados, campos arrasados y soldados o guerrilleros que enseñan sus armas. La idea de que esta evocación permitirá la consolidación nacional, y con ella la convivencia pacífica, se convierte en un recurso rebasado por el carácter catastrófico de los hechos narrados.

La recurrencia y la abundancia en la escritura de textos sobre la violencia en Colombia evidencian un afán de encontrar en el lenguaje una alternativa social a la misma. El discurso sobre la violencia puede ser sin embargo problemático, porque tiende a toparse con un territorio en el que reina el silencio, donde se encuentran los muertos causados por el objeto de estudio. Ese mismo silencio puede entenderse también, sin embargo, como un impulso que alienta el deseo de elaborar una escritura al respecto. Se trata de un proceso con múltiples implicaciones políticas, jurídicas, éticas y estéticas. La exploración de estas implicaciones, su presencia e importancia, y en especial su relación con los hechos violentos, son un campo abierto para muchas reflexiones que resultan necesarias en una época en la cual la violencia aparece cada vez más como un fenómeno incomprensible, temible e inabarcable. Paradójicamente, asumir la incapacidad del discurso para deshacer los actos de violencia puede ser un camino para entender su acción, señalar la responsabilidad de quienes los promueven y minimizar el daño que causan.

BiBLIOGRAFÍA

Anderson, Benedict. Imagined Communities. Londres: Verso, 1991.

Arocha, Jaime y Gonzalo Sánchez. Colombia, violencia y democracia. Bogotá: Universidad Nacional de Colombia, Centro Editorial, 1987.

Bhabha, Homi. "DissemiNation". Nation and Narration. Homi Bhabha, ed. Londres: Routledge, 1990. 291-320. 
Camacho, Álvaro, Álvaro Guzmán, María Clemencia Ramírez y Fernando Gaitán. Nuevas visiones sobre la violencia en Colombia. Bogotá: Fescol-IEPRI, 1997.

Colmenares, Germán. Las convenciones contra la cultura. Ensayos sobre historiografía hispanoamericana del siglo XIX. Bogotá: Tercer Mundo Editores, 1986.

De Certeau, Michel. L'écriture de l'histoire. París: Editions Gallimard, 1975.

Guzmán, Germán, Orlando Fals Borda y Eduardo Umaña Luna. La violencia en Colombia. Estudio de un proceso social. Bogotá: Ediciones Tercer Mundo, 1962.

Henao, Jesús María y Gerardo Arrubla. Historia de Colombia para la enseñanza secundaria. 2 vols. Bogotá: Escuela Tipográfica Salesiana, 1912.

Melo, Jorge Orlando. "La evolución económica de Colombia, 1830-1900”. Manual de Historia de Colombia. Jaime Jaramillo Uribe, et al. Tomo II. Bogotá: Instituto Colombiano de Cultura, 1979. 133-207.

Palacios, Marco y Frank Safford. Colombia: país fragmentado, sociedad dividida. Bogotá: Grupo Editorial Norma, 2002.

Pécault, Daniel. Guerra contra la sociedad. Bogotá: Planeta, 2001.

Rama, Ángel. La ciudad letrada. Hanover: Ediciones del Norte, 1984.

Rojas, Cristina. Civilization and Violence. Regimes of Representation in Nineteenth-Century Colombia. Minneapolis: U of Minnesota P, 2002.

Sánchez, Gonzalo y Donny Merteens. Bandoleros, gamonales y campesinos. Bogotá: El Áncora Editores, 1983.

Suárez Rondón, Gerardo. La novela sobre la violencia en Colombia. Bogotá: Ediciones Luis F. Serrano, 1966.

Uribe, María Victoria. Matar, rematar y contramatar. Bogotá: CINEP, 1996.

Villaveces Izquierdo, Santiago. "The Crossroads of Faith: Heroism and Melancholia in the Colombian 'Violentologists' (1980-2000)”. Cultural Agency in the Americas. Doris Sommer, ed. Durham: Duke UP, 2006. 305-25. 